http://jmscr.igmpublication.org/home/ ISSN (e)-2347-176x ISSN (p) 2455-0450

crossref DOI: https://dx.doi.org/10.18535/jmscr/v7i11.66

\title{
Comparison of Sonohysterosalpingography Using Agitated Saline and Hysterosalpingography to Evaluate Tubal Patency in Infertile Women
} Authors

\section{Dr Tejaswi Chalumuri MD (RD)*, Dr Dinesh Chalumuri MD (RD), Dr Y. Trinadh}

Department of Radio-Diagnosis, Andhra Medical College, Visakhapatnam, India

*Corresponding Author

\section{Dr Tejaswi Chalumuri MD (RD)}

\begin{abstract}
Background: Sonohysterosalpingography (SonoHSG) is a new method for assessing tubal patency using transvaginal ultrasound. It is having several advantages over conventional hysterosalpingography (HSG). The present study prospectively evaluated the performance of SonoHSG and HSG in establishing tubal patency or blockage.

Materials and Methods: This prospective observational study was carried out to assess the value of sonohysterosalpingography (SonoHG) in evaluating tubal patency in infertile patients and to compare its results with hysterosalpingography (HSG) and laparoscopic chromotubation. This study included 54 infertile women. All women underwent both SonoHSG and HSG. Patients in whom tubal block had been established by either SonoHSG or HSG underwent laparoscopic chromotubation. Each tube was evaluated individually.

Results: With laparoscopic findings as the gold standard, sonohysterosalpingography had a sensitivity of $100 \%$, specificity of $85.7 \%$, positive predictive value of $87.5 \%$ and a negative predictive value of $100 \%$. The rate of agreement with laparoscopy was $87.5 \%$. The findings for the same parameters using HSG were $100 \%, 75 \%, 80 \%$, and 100\%, respectively. The rate of laparoscopic agreement with HSG was $8 \%$. Sonohysterosalpingography showed more uterine abnormalities than HSG.

Conclusion: Sonohysterosalpingography using agitated saline is a low-cost, reliable, safe examination method. It can be used for the primary investigation of infertility on an outpatient basis. When performed by experienced operators, it serves as a valuable, first-line screening test for more invasive procedures like laparoscopic chromotubation.
\end{abstract}

\section{Introduction}

Infertility is defined as the failure to conceive after 1year of regular unprotected intercourse. The major causes of infertility include ovulatory dysfunction (15\%), tubal and peritoneal pathology (30 to $40 \%$ ), and male factors (30 to $40 \%$ ).

Tubal disease is one of the most frequent causes of infertility, occurring in up to $30 \%$ of infertile women ${ }^{1}$. Demonstration of tubal patency can allow less invasive treatment. There are several tests available for this purpose, including traditional tests like hysterosalphingography (HSG), laparoscopy (LSC). Though LSC is gold standard for tubal assessment, it is associated with anaesthetic and surgical risks. HSG has disadvantages of requiring radiography equipment, gonadal exposure to radiation and hypersensitivity to iodine dye, used as contrast for the test. Hence, there is a need for alternative methods of tubal patency tests. As a result, an 
ultrasound technique using agitated saline has emerged. Agitated saline has advantage over saline as medium, as air micro-bubbles create scintillating echoes on ultrasound allowing clear visualisation of the tubal spillage and fallopian tubes ${ }^{1}$.

The purpose of this study was to establish the role of sonohysterosalpingography using agitated saline in evaluating tubal patency and comparing its efficacy to hysterosalpingography in Indian population.

\section{Materials and Methods}

This prospective observational study was carried out in the Departments of Radiodiagnosis and Obstetrics \& Gynaecology, Andhra Medical College, Visakhapatnam. A total of 54 women attending the infertility clinic of the department of Obstetrics \&Gynaecology from December 2016 to April 2018 formed the study group.

The sample size of 54 was estimated by statistical formula, with an expected difference of sensitivity in Sono-HSG and HSG with laparoscopy as $14 \%$ $(99 \% \mathrm{Vs} 85 \%)$ at $5 \%$ level of significance and $80 \%$ power. The protocol of the study was approved by the research council and ethical committee of the institute. Informed consent was taken from all patients who participated in the study.

\section{Inclusion Criteria}

1. Women between age group 20 to 40 years presenting with primary and secondary infertility.

\section{Exclusion Criteria}

1. Suspected pregnancy.

2. Reproductive tract cancer.

3. Endocrine disorders like thyroid and pituitary disorders.

4. Contraindication for contrast study
a) Allergy to iodine agents,
b) Asthma,
c) severe renal dysfunction,
d) dehydration
e) Active pelvic inflammatory disease.

\section{Results}

The present study included 54 subjects, with age wise distribution of the study inclusions as follows:

Majority (48\%) of the women were in 25-29 age group. Fourteen $(25.9 \%)$ of them were in the 20 24 year group. Ten women (18.5\%) belonged to 30-34 age group. A small proportion of the women, 4 in number $(7.4 \%)$ belonged to greater than 35 years age group. The mean age of patients was 27 years.

Figure 1: Duration of Infertility

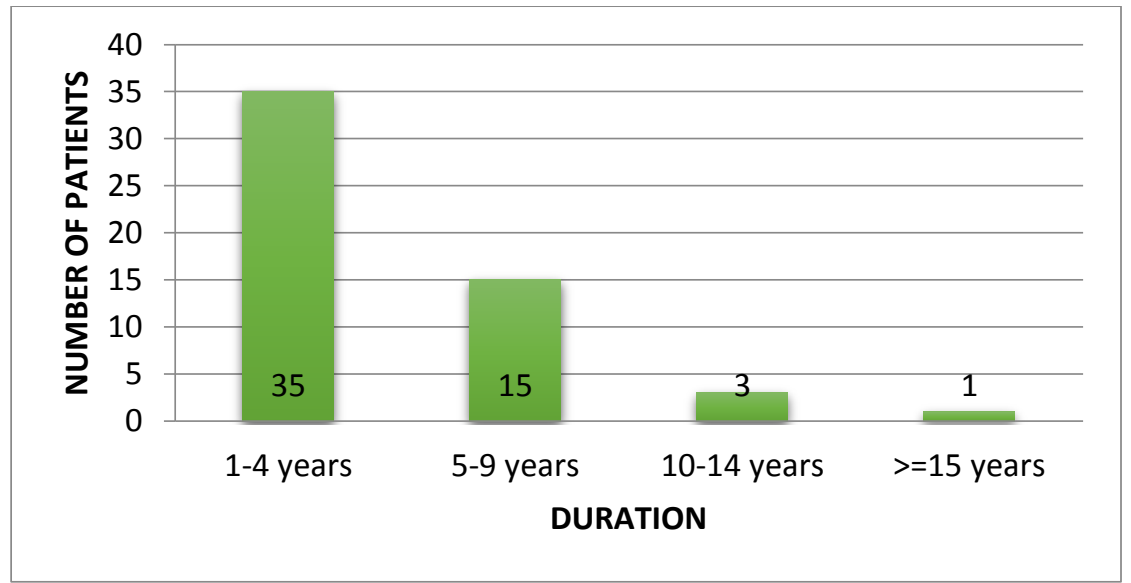

In majority, 35 women $(64.8 \%)$ the duration of infertility was $1-4$ years. Fifteen women $(27.7 \%)$ were infertile for 5-9 years. Three women $(5.5 \%)$ were infertile for 10-14 years. Only a single woman $(1.8 \%)$ was infertile for more than fifteen years. The mean duration of infertility was 4.1years. 
Figure 2: Infertility status

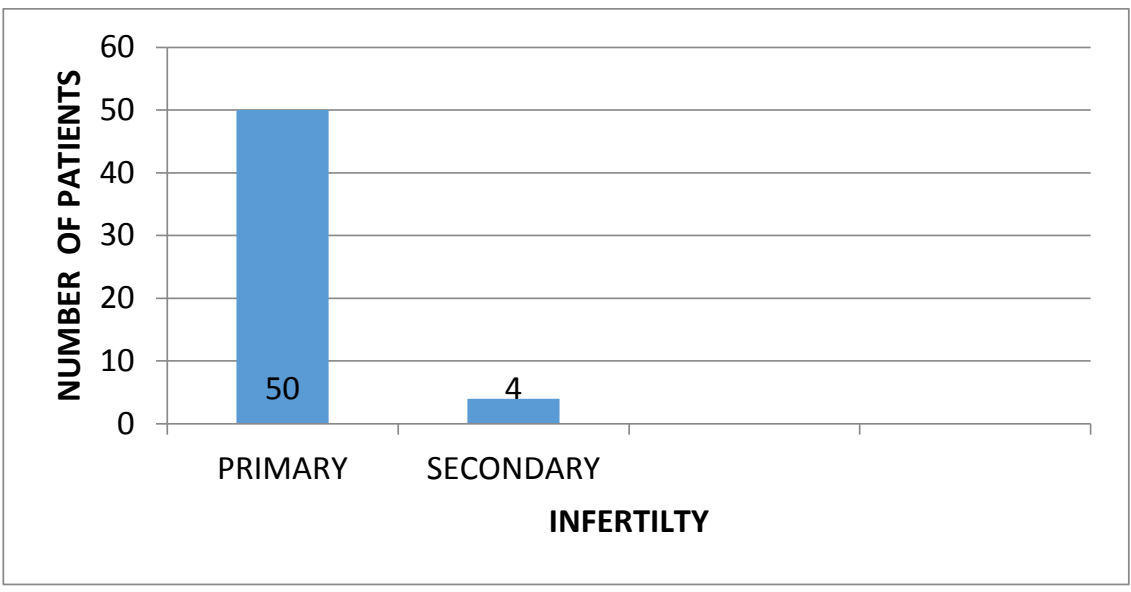

Majority (92.6\%) of women were of primary infertility. Four (7.4\%) women belonged to secondary infertility group.

Table 1: Comparison of Sono HSG and HSG in detecting uterine, ovarian and other abnormalities $(n=54)$.

\begin{tabular}{|l|c|c|c|}
\hline & Uterine & Ovarian & $\begin{array}{c}\text { Other } \\
\text { Abnormalities }\end{array}$ \\
\hline SONO HSG & 5 & 3 & none \\
\hline HSG & 1 & $*$ & 1 \\
\hline
\end{tabular}

\section{*=cannot be assessed}

Sono HSG showed more uterine abnormalities than HSG. Sono HSG showed fibroids in three women, in which one of them had multiple fibroids. Adenomyosis was noted in one patient and polyp noted in one patient. Ovarian abnormalities were noted in 3 patients, in which two showed PCOD and the other showed ovarian cyst with a probability of endometrioma. HSG showed fibroid only in one patient and peritubal adhesions in one patient, which was later confirmed in laparoscopy

Table 2: Sono HSG tubal patency results. Number of tubes $(n=108)$

\begin{tabular}{l|c|}
\hline RESULT & NUMBER OF TUBES(n=108) \\
\hline Patent & $100(92.6 \%)$ \\
\hline Bilateral block & $* 2(1.8 \%)$ \\
\hline Left block & $4(3.7 \%)$ \\
\hline Right block & $2(1.8 \%)$ \\
\hline *bilateral block in one woman, so number of tubes \\
blocked were two tubes.
\end{tabular}

As the total number of patients showing tubal blocks are less, thereby reducing the number of women undergoing laparoscopy, number of tubes have been considered for the statistical analysis. Patency was seen in 100 tubes $(92.6 \%)$. Bilateral block in one woman, that was two tubes $(1.8 \%)$. Left block was noted in 4 women (3.7\%) and right block was noted in 2 women $(1.8 \%)$.

Figure 3: HSG tubal patency results

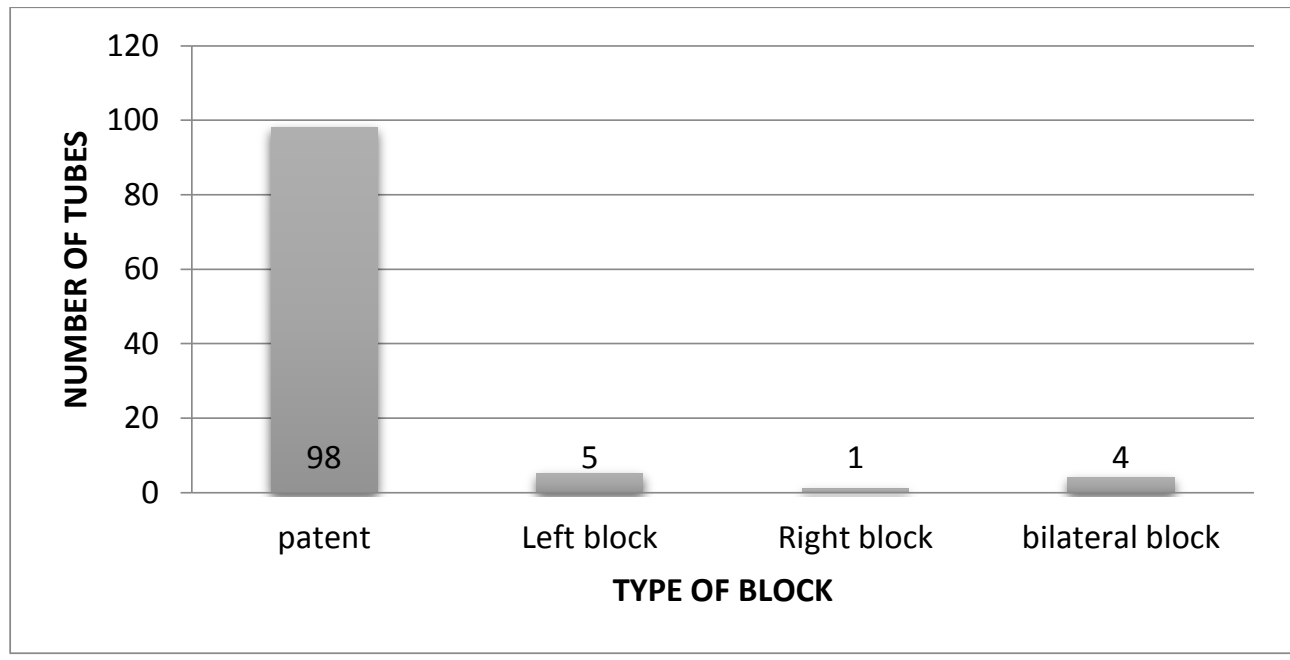


Patency was seen in 98 tubes $(90.7 \%)$. Bilateral block was noted in two patients, which were 4 tubes $(3.7 \%)$. Left block was noted in 5 women $(4.6 \%)$ and right block noted in 1 woman $(0.9 \%)$.
Table 3: Laparoscopic results for Sono HSG blocked tubes $(\mathrm{n}=8)$

\begin{tabular}{|c|c|c|}
\hline & Sono HSG & Laparoscopy \\
\hline Right block & 2 & $1(14.28 \%)$ \\
\hline Left block & 4 & $4(57.14 \%)$ \\
\hline Bilateral block & $2 *$ & $2(28.57 \%)$ \\
\hline
\end{tabular}

Figure 4: Laparoscopic results for Sono HSG blocked tubes $(n=8)$

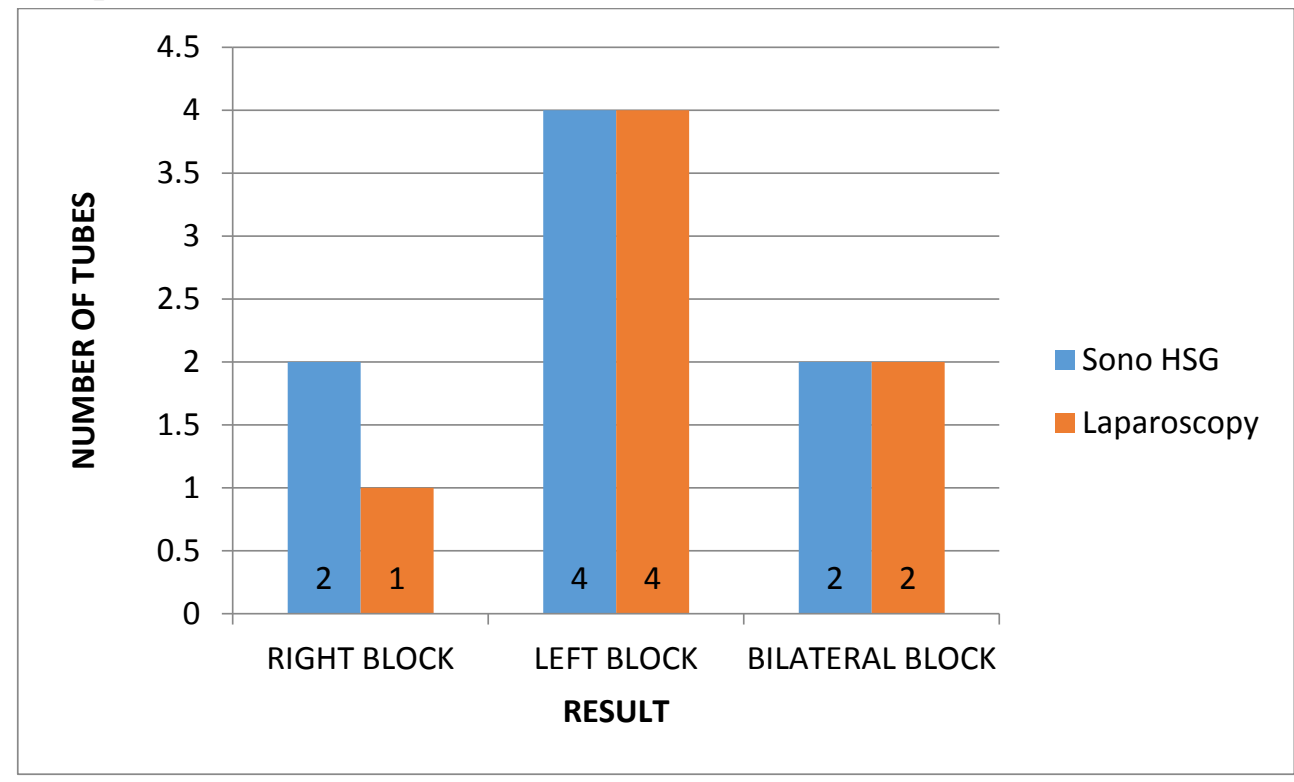

Laparoscopy found right block only in one tube (14.28\%), while Sono HSG showed two blocked tubes. Left block was seen in 4 tubes $(57.14 \%)$ confirming the findings in Sono HSG. Bilateral block was seen in one woman in Sono HSG, so number of tubes blocked were 2, which was confirmed in laparoscopy. The rate of agreement was $87.5 \%$ (7/8) when individual tubes were considered. There was a single case of discordance, the reason for which could be tubal spasm.

Table 4: Laparoscopic results for HSG blocked tubes $(n=10)$

\begin{tabular}{|l|c|c|}
\hline & HSG & Laparoscopy \\
\hline Right block & 1 & $1(12.5 \%)$ \\
\hline Left block & 5 & $5(62.5 \%)$ \\
\hline Bilateral block & $* 4$ & $2(31.25 \%)$ \\
\hline
\end{tabular}

* Bilateral block was in two patients, so number of blocked tubes were four.

Laparoscopy found right block in 1 tube (12.5\%). Left block was seen 5 tubes $(62.5 \%)$. Bilateral block was seen in two women in HSG, so number of tubes blocked were 4 . The rate of agreement was $80 \%(8 / 10)$ when individual tubes were considered. Discordance was seen in two cases, the reason for which could be tubal spasm.

Table 5: Statistics of tubal patency testing by sono hysterosalpingography Vs laparoscopy for individual tubes. Statistics were evaluated for seven patients who underwent laparoscopy

\begin{tabular}{|c|c|c|c|}
\hline Sensitivity & Specificity & $\begin{array}{c}\text { Positive } \\
\text { predictive } \\
\text { value }\end{array}$ & $\begin{array}{c}\text { Negative } \\
\text { predictive } \\
\text { Value }\end{array}$ \\
\hline $100 \%$ & $85.7 \%$ & $87.5 \%$ & $100 \%$ \\
\hline
\end{tabular}

Table 6: Statistics of tubal patency testing by hysterosalpingography Vs laparoscopy for individual tubes. Statistics were evaluated for eight patients who underwent laparoscopy

\begin{tabular}{|l|c|c|c|}
\hline Sensitivity & Specificity & $\begin{array}{c}\text { Positive } \\
\text { predictive } \\
\text { value }\end{array}$ & $\begin{array}{c}\text { Negative } \\
\text { predictive } \\
\text { Value }\end{array}$ \\
\hline $100 \%$ & $75 \%$ & $80 \%$ & $100 \%$ \\
\hline
\end{tabular}




\section{Discussion}

Hysterosalpingography is still the primary investigation to assess tubal patency in infertility cases in most centresin South East Asia. It is however, time consuming, labour intensive and carries risk of reaction to contrast media and involves radiation exposure. Laparoscopy is required if tubal surgery is contemplated or other causes of infertility are to be excluded. Although laparoscopy is considered to provide the most reliable method of assessing tubal patency before surgery, it does not provide information about the cavity of the uterus and fallopian tubes. It is also invasive, requires anaesthesia and needs hysteroscopy to visualise the uterine cavity.

Studies like Volpi et $\mathrm{al}^{4}$ and Jeany et $\mathrm{al}^{2}$ has used air and saline separately, injecting either one of them following the other. The present study used agitated saline as contrast media in sonohysterosalpingography, same as Chenia et $\mathrm{al}^{3}$ study, where in air and saline are mixed and injected together. No problems were encountered due to agitated saline in the interpretation of the sonographic images.

Volpi et $\mathrm{al}^{4}$ in a prospective study involving 154 women, reported that the average duration of infertility was 1.7 years and that the average age at infertility was 31.7 years ${ }^{4}$. Jeanty et al. study conducted in 115 women found that the average duration of infertility was 2.2 years and the average age at infertility was 31.9 years ${ }^{2}$. In the present study the average duration of infertility was higher, recording at 4.1 years, while the average age at infertility was lower than the other studies, recording at 27 years.

Jeanty et $\mathrm{al}^{2}$. study conducted in 115 women found that sonohysterosalpingography allows identification of uterine and ovarian abnormalities that are relevant to management of patients with infertility - like polyps,fibroids, $\mathrm{PCOD}^{2}$. In their study, three patients had fibroids, including two in whom the fibroids were submucosal. Nine patients had one or more polyps within the uterine cavity, and three patients had synechiae. The present study supports their observation. Sono HSG showed more uterine abnormalities than HSG. It showed fibroids in three women, in which one of them had multiple fibroids; adenomyosis was noted in one patient and polyp in one patient. Ovarian abnormalities were noted in 3 patients, in which two showed PCOD and the other showed ovarian cyst with a probability of endometrioma. Five patients had cervical stenosis which prevented the procedure in the study of Jeanty et $\mathrm{al}^{2}$, while none were encountered in the present study.

A number of investigators have evaluated the efficacy of sonohysterosalpingography and hysterosalpingography in determining tubal patency. Chenia et al. in their study in 71 women found sonohysterosalpingography was in agreement with hysterosalpingography in $85 \%$ of the cases ${ }^{3}$. Jeanty et al assessed the use of air as a sonographic contrast agent in the investigation of tubal patency by sonohysterosalpingography in a group of 115 infertile women and found airsonohysterosalpingography and laparoscopy showed agreement in $79.4 \%{ }^{2}$. Volpi et al in their sonohyterosalpingography study in 154 patients using air-saline as contrast media showed laparoscopic agreement in $89.3 \%$. In the present study the rate of agreement was $87.5 \%$ between sonohysterosalpingography and laparoscopy, which is almost similar to Volpi et $\mathrm{al}^{4}$ and Chenia et $\mathrm{al}^{3}$ studies. Hyterosalpingography and laparoscopy showed a rate of agreement of $80 \%$ in the present study.

Volpi et $\mathrm{al}^{4}$ SonoHSG study in 154 women revealed bilateral tubal patency in 106 cases $(68.8 \%)$, of which $34(22.1 \%)$ cases showed unilateral tubal occlusion and $13(8.4 \%)$ with bilateral tubal occlusion. In one case it was not possible to assess the tubal patency. The present study was of a smaller sample size compared to the above study and was in 54 women. It showed bilateral tubal patency in 47 women $(87 \%)$, unilateral tubal occlusion in 6 women $(11.1 \%)$ and bilateral block in one patient $(1.85 \%)$.

Chenia et $\mathrm{al}^{3} \mathrm{in}$ their study of 142 individual fallopian tubes, 120 tubes were patent in both 
SonoHSG and HSG. Laparoscopic information was available in 15 women. The findings in SonoHSG and HSG were in agreement with respect to 19 of the tubes studied laparoscopically. The results differed for 11 tubes. Laparoscopy confirmed SonoHSG diagnosis in 7 tubes, HSG diagnosis in 3 tubes and neither modality for one tube. In the present study, considering that 108 individual tubes were studied, SonoHSG showed patency in 100 tubes(92.6\%), bilateral block in one woman - i.e two tubes $(1.8 \%)$, left block in 4 women(3.7\%) and right block in 2 women(1.8\%). Laparoscopy found right block only in one tube(14.28\%), among two blocked tubes. Left block was seen in 4 tubes $(57.14 \%)$, confirming the findings in SonoHSG. Bilateral block seen in SonoHSG was confirmed in laparoscopy. False positive results in sonohysterosalpingography and hysterosalpingography in the present study could probably have occurred due to tubal spasm. A second SonoHSG examination can rule out tubal spasm before the patient undergoes laparoscopy. Since the test is repeatable without impairment of reproductive potential, SonoHSG can be performed twice before resorting to another classical tubal patency test.

The sensitivity, specificity, positive predictive value and negative predictive value for sonohysterosalpingography in Jeanty et $\mathrm{al}^{2}$ study was $87.5 \%, 80.9 \%, 63.6 \%$ and $94.4 \%$ respectively, while Volpi et $\mathrm{al}^{3}$ study showed $80 \%, 85 \%, 80 \%$ and $85 \%$ respectively. The present study showed superior results. The sensitivity, specificity, positive predictive value and negative predictive value for sonohysterosalpingography in the present study was $100 \%, 85.7 \%, 87.5 \%$ and $100 \%$ and for hysterosalpingography, it was $100 \%, 75 \%, 80 \%$ and $100 \%$ respectively. While the sensitivity and negative predictive value of both sonohysterosalpingography and hysterosalpingography are the same, specificity and positive predictive value of sonohysterosalpingography appears to be superior in the present study. However, this comparison should be treated cautiously as laparoscopic results are not available in the entire study group as it was done only when either of the modalities showed tubal block.

There were no significant complications in the present study except for abdominal cramping, which was relieved with administration of analgesics, whereas, Inki et $\mathrm{al}^{5}$ and Jeanty et $\mathrm{al}^{2}$ each showed a case of vasovagal reaction apart from minor complications like abdominal cramping and shoulder pain.

Ultrasound examination of the fallopian tubes is well established in gynaecological practice, particularly when gross pathology such as ectopic pregnancy, hydrosalpinx or tubo-ovarian abscess is present. Evaluation of healthy fallopian tubes is difficult as normal tube is a poor sonic reflector devoid of the tissue/fluid interfaces that would produce a clear organ outline. Chenia et $\mathrm{al}^{2}$. found that the use of agitated saline improved the ease with which the fallopian tubes were visualised. In present study it was found that demonstration of cornual and fimbrial end of tubes was easy, while demonstration of isthmic portion of tubes was difficult.

\section{Conclusion}

In conclusion, this study demonstrated good agreement with the gold standard (laparoscopic chromotubation) in $87.5 \%$ of the tubes. Sonohysterosalpingography with agitated saline is an inexpensive, relatively fast, well tolerated, safe method to assess the fallopian tube patency in cases of infertility.

Tubal patency testing by sonohysterosalpingography can be implemented routinely using agitated saline, thereby reducing the number of unnecessary laparoscopies performed on infertile patients due to the current standard diagnostic approach of combining HSG and laparoscopy. Avoiding complications related to laparoscopy may thus be achieved, and such a policy would also lead to reduction in health care costs in the management of infertility. 


\section{References}

1. Volpi E, Zuccaro, Patriarca P. Transvaginal sonographic tubal patency testing using air and saline solution as contrast media in a routine infertility clinic setting. Ultrasound Obstet. Gynecol 1998;7:43-48.

2. Agur, Anne M.R.; Dalley, Arthur F;pelvis and perineum In:Grant's Atlas of Anatomy.12th Edition. Lippincott Williams \& Wilkins 2009;236-38.

3. Philippe Jeanty, Besnard S, Arnold A, Turner C, Crum P. Air-Contrast Sonohysterography as a First Step Assessment of Tubal Patency. J Ultrasound Med 2000; 19:519-527.

4. Chapron C, Querleu D, Bruhat MA, et al. Surgical complications of diagnostic and operative gynaecological laparoscopy: a series of 29,966 cases. Hum Reprod 1998;13:867-872.

5. Leinkauf-Houcken A, Huneken B, Lindner $\mathrm{C}$, et al: Combining B-mode ultrasound with pulsed wave Doppler for the assessment of tubal patency. Hum Reprod 1997;12:2457-2960. 